\title{
Durability of Membrane Electrode Assemblies (MEAs) in PEM Fuel Cells Operated on Pure Hydrogen and Oxygen
}

\author{
Vesna Stanic, James Braun \\ Teledyne Energy Systems, West Palm Beach, FL, 33417 \\ Mark Hoberecht \\ NASA Glenn Research Center, Cleveland, OH, 44135
}

\begin{abstract}
Proton exchange membrane (PEM) fuel cells are energy sources that have the potential to replace alkaline fuel cells for space programs. Broad power ranges, high peak-to-nominal power capabilities, low maintenance costs, and the promise of increased life are the major advantages of PEM technology in comparison to alkaline technology. The probability of PEM fuel cells replacing alkaline fuel cells for space applications will increase if the promise of increased life is verified by achieving a minimum of 10,000 hours of operating life. Durability plays an important role in the process of evaluation and selection of MEAs for Teledyne's Phase I contract with the NASA Glenn Research Center entitled "Proton Exchange Membrane Fuel cell (PEMFC) Power Plant Technology Development for $2^{\text {nd }}$ Generation Reusable Launch Vehicles (RLVs)". For this contract, MEAs that are typically used for $\mathrm{H}_{2} /$ air operation were selected as potential candidates for $\mathrm{H}_{2} / \mathrm{O}_{2}$ PEM fuel cells because their catalysts have properties suitable for $\mathrm{O}_{2}$ operation. They were purchased from several well-established MEA manufacturers who are world leaders in the manufacturing of diverse products and have committed extensive resources in an attempt to develop and fully commercialize MEA technology. A total of twelve MEAs used in $\mathrm{H}_{2}$ /air operation were initially identified from these manufacturers. Based on the manufacturers' specifications, nine of these were selected for evaluation.
\end{abstract}

Since 10,000 hours is almost equivalent to 14 months, it was not possible to perform continuous testing with each MEA selected during Phase I of the contract. Because of the lack of time, a screening test on each MEA was performed for 400 hours under accelerated test conditions. The major criterion for an MEA pass or fail of the screening test was the gas crossover rate. If the gas crossover rate was higher than the membrane intrinsic permeability after 400 hours of testing, it was considered that the MEA had failed the test. Three types of MEAs out of the nine total membranes failed the test.

The evaluation results showed that fuel cell operating conditions (current, pressure, stoichiometric flow rates) were the parameters that influenced the durability of MEAs. In addition, the durability test results indicated that the type of membrane was also an important parameter for MEA durability. At accelerated test conditions, the MEAs with casted membranes failed during the 400 hour test. However, the MEAs prepared from the casted membrane with support as well as extruded membranes, both passed the $400 \mathrm{~h}$ durability test at accelerated operating test conditions.

As a result of the MEA accelerated durability tests, four MEAs were selected for further endurance testing. These tests are being carried out with four-cell stacks under nominal fuel cell operating conditions. 


\title{
DURABILITY OF MEMBRANE ELECTRODE ASSEMBLIES (MEAS) IN PEM FUEL CELLS OPERATED ON PURE HYDROGEN AND OXYGEN
}

\author{
Vesna Stanic, James Braun \\ Teledyne Energy Systems, West Palm Beach, FL, 33417 \\ Mark Hoberecht \\ NASA Glenn Research Center, Cleveland, OH, 44135
}

\begin{abstract}
$\underline{\text { Abstract }}$
Durability played an important role in the process of evaluation and selection of MEAs for Teledyne's Phase I contract with the NASA Glenn Research Center entitled "Proton Exchange Membrane Fuel cell (PEMFC) Power Plant Technology Development for Next Generation Launch Technology (NGLT)". For this contract, MEAs that are currently available on the market for $\mathrm{H}_{2}$ /air operation were selected as potential candidates for $\mathrm{H}_{2} / \mathrm{O}_{2}$ PEM fuel cells. They were purchased from several well-established MEA manufacturers.
\end{abstract}

A screening test on each MEA was performed for 400 hours under accelerated test conditions. The major criterion for an MEA pass or fail of the screening test was the gas crossover rate. If the gas crossover rate was higher than the membrane intrinsic permeability after 400 hours of testing, it was considered that the MEA had failed the test. The objectives of the MEA accelerated durability test were to select MEAs for further endurance testing and to identify the test conditions that will enable the MEA extended life.

The evaluation results showed that not only temperature and humidity, but also current, pressure, and gas stoichiometry influence the MEA durability. In addition, the durability test results indicated that the type of membrane was also important. At accelerated test conditions, the MEAs with casted membranes failed during the 400 hour test. However, both the MEAs prepared from both the casted membrane with a reinforcement as well as the extruded membranes, passed the 400 hour durability test at accelerated operating test conditions.

\section{Introduction}

Proton exchange membrane (PEM) fuel cells are energy sources that have the potential to replace alkaline fuel cells for space programs. Broad power ranges, high peak-to-nominal power capabilities, low maintenance costs, and the promise of increased life are the major advantages of PEM technology in comparison to the alkaline. The probability of PEM fuel cells replacing alkaline fuel cells for space applications will increase if the promise of increased life is verified by achieving a minimum of 10,000 hours of operating life.

Since the MEA is the most critical component of PEM fuel cells, MEA durability evaluation played an important role in Teledyne's Phase I contract with the NASA Glenn Research Center entitled "Proton Exchange Membrane Fuel cell (PEMFC) Power Plant Technology Development for Next Generation Launch Technology (NGLT)".

For this contract, MEAs that are typically used for $\mathrm{H}_{2} /$ air operation were selected as potential candidates for $\mathrm{H}_{2} / \mathrm{O}_{2}$ PEM fuel cells because their catalysts have properties suitable for $\mathrm{O}_{2}$ operation. They were purchased from several well-established MEA manufacturers who are world leaders in MEA manufacturing and have committed extensive resources in an attempt to develop and fully commercialize MEA technology. A total of twelve MEAs used in $\mathrm{H}_{2}$ /air operation were initially identified from these manufacturers, and nine of these were selected for evaluation.

In this paper, the MEAs that were tested had different membrane formulations, catalyst composition, and gas diffusion layers (GDLs). They were tested in 4-cell NG 2000 fuel cell stacks with hydrogen and oxygen for 400 hours. The 400 hour duration screening test was established with a series of experiments in which cell current, gas stoichiometries, and pressure were changed while keeping fuel cell temperature and humidification constant. The major criterion for an MEA to pass or fail the screening test was the gas crossover rate. Gas crossover was checked approximately every 100 hours of stack run time. The failure modes of MEAs were analyzed by optical and scanning electron microscopes. 


\section{Experimental}

MEAs selected for durability evaluation

The nine MEAs selected for durability evaluation are listed in Table 1. Even though the membranes of MEAs were made of the same type of polymer $(\sim 1100 \mathrm{EW}$ persulfonated tetraflouroethylene) they differed in thickness and processing. The hydrogen and oxygen electrocatalyst in all MEAs was platinum supported on carbon.

GDLs used with the MEAs differed in the type of carbon fiber material used, their processing, micro diffusion layer composition, and thickness.

Table 1: The MEAs selected for evaluation in NG2000 fuel cells with $\mathrm{H}_{2} / \mathrm{O}_{2}$. Different MEAs are labeled with numbers 1 through 9 while. Membrane thickness is generically presented with capital letter $\mathrm{T}$ and a number. The thinnest membrane is labeled with $\mathrm{T}$, while the thickest with $5 \mathrm{~T}$.

\begin{tabular}{lll}
\hline MEA & MEMBRANE THICKNESS $(\mu \mathrm{m})$ & MEMBRANE TYPE \\
\hline 1 & $\mathrm{~T}$ & Casted, polymer only \\
2 & $2 \mathrm{~T}$ & Casted, polymer only \\
3 & $2 \mathrm{~T}$ & Extruded, polymer only \\
4 & $3 \mathrm{~T}$ & Extruded, polymer only \\
5 & $4 \mathrm{~T}$ & Extruded, polymer only \\
6 & $5 \mathrm{~T}$ & Extruded, polymer only \\
7 & $2 \mathrm{~T}$ & Extruded, polymer only \\
8 & $\mathrm{~T}$ & Casted, polymer with reinforcement \\
9 & $2 \mathrm{~T}$ & Extruded, polymer only \\
\hline
\end{tabular}

\section{MEA test procedure}

Selected MEAs were tested in a 4-cell NG 2000 fuel cell stack with hydrogen and oxygen. Before the durability evaluation started, six fuel cell tests were performed using MEA1 in order to define the operating conditions for controlling MEA durability (accelerated failure or extended life). The operating conditions varied were fuel cell current, the reactant gas stoichiometries, and pressures. The stack operating temperature and humidification remained constant during all tests, $60^{\circ} \mathrm{C}$ and $100 \% \mathrm{RH}$. Based on the MEA endurance, two different sets of operating conditions were defined, "accelerated" and "optimal." At accelerated test conditions, MEAs failed within 400 hours. However, optimal conditions facilitated MEAs to last more than 1000 hours.

The only tests performed at accelerated conditions were MEA durability screening. A criterion used for an MEA to pass or fail the test was the gas crossover rate. When the gas crossover rate was higher than the MEA intrinsic permeability, it was considered that the MEA failed the test.

The intrinsic permeability of a membrane is a natural process that is driven by gas diffusion. The gas diffusion depends on gas concentration (pressure) gradient and diffusion constant, as defined by Fick's laws [1]. On the other hand, the diffusion constant depends on the membrane properties, the size of gas molecules, and temperature. Thus, the intrinsic permeability of a membrane increases if measured at higher temperature $\left(60^{\circ} \mathrm{C}\right.$ vs. $\left.25^{\circ} \mathrm{C}\right)$ and higher differential pressure ( 20 psi vs. 5 psi), and with hydrogen instead of nitrogen with a fully humidified membrane. A typical hydrogen intrinsic permeability for fully humidified polymer exchange membranes [2] is $-17 \times 10^{-5} \mathrm{~cm}^{3} / \mathrm{s} \cdot \mathrm{cm}^{2}$ at 5 psid and $25^{\circ} \mathrm{C}$. The value recalculated for nitrogen is $3.3 \times 10^{-5} \mathrm{~cm}^{3} / \mathrm{s} \cdot \mathrm{cm}^{2}$ and it was used as an intrinsic permeability limit for all MEAs tested.

In addition, polarization curves were generated to evaluate a full range of the MEA performance.

\section{Gas crossover test}

The gas crossover is measured using a gas leak check fixture designed for stack overboard leak and crossover measurements. The measurements are performed on a non operational stack with fully humidified MEAs at room temperature with nitrogen at 5 psid. The gas crossover is checked at every 100 hours of run time. 
Optical and Scanning electron microscopes

These methods are used for the examination of the MEA failure modes. The examination is carried out with an Olympus BX60 optical microscope in different light modes and at different magnifications. The magnification range of this optical microscope is from 50 to 1000 times. For MEAs and GDL investigations at higher magnifications, a Cambridge Instruments Stereoscan 120 scanning electron microscope is used. $\underline{\text { Results }}$

\section{Definition of Accelerated and Nominal operating conditions}

The purpose of six tests performed at different operating conditions was to determine the set of conditions that will cause the accelerated but controlled failure of an MEA 1. Temperature and relative humidity were constant while gas stoichiometry, current density, and pressure were varied during these tests.

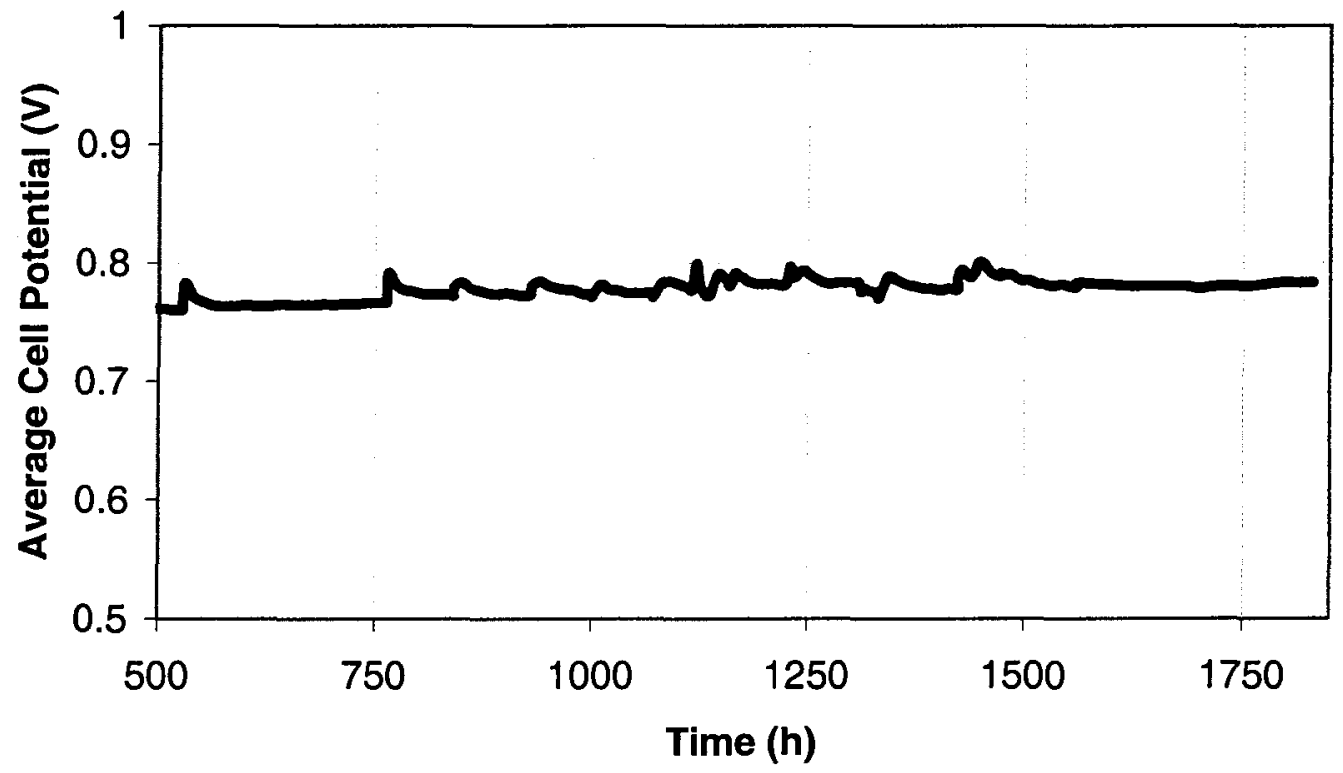

Figure 1: Endurance run of MEA 1 at nominal operating conditions in NG 2000 fuel cell with hydrogen and oxygen at $60^{\circ} \mathrm{C}$ and $100 \% \mathrm{RH}$.

The set of conditions that caused the MEAs to fail within 400 hours was identified as "accelerated" and was used for the MEA durability screening. In addition, the nominal operating conditions were also identified. At these conditions, MEA 1 ran for more than 1300 hours in hydrogen/oxygen without failure, as presented in Figure 1.

These results demonstrated that gas stoichiometry, pressure and current have a big influence on MEA durability. The optimization of these parameters can extend the MEA life time in PEM fuel cells.

\section{MEA durability}

Three MEAs out of nine failed the 400 hour durability screening test. These MEAs are 1, 2, and 7. All other MEAs tested $(3,4,5,6$, and 8$)$ passed the test.
MEAs 1 and 2 have polymer electrolyte membranes produced by polymer casting, while MEAs 3, 4, 5, 6 and 9 membranes were manufactured using a polymer extrusion process. The MEA failures were identified and monitored by measuring the gas crossover rates. The results are presented in Figure 2. The graphs show that all three MEAs had constant gas crossover at the beginning of the test, equivalent to the membrane intrinsic permeabilities. In addition, the graphs demonstrate that the intrinsic permeability of MEA 1 membrane is higher than that of either MEA 2 or 7. This result is in agreement with previous studies that showed that membrane permeability decreases as the thickness increases.

Once the gas crossover occurred, its increase over time was a function of the severity of MEA damage. MEAs 
1 and 2 had holes ("hot spots") that developed slowly over time as shown in Figure 2. After a $\sim 400$ hour test with oxygen, the gas crossover of MEA 1 was $2 \times 10^{-5}$ $\mathrm{cm}^{3} / \mathrm{s} \cdot \mathrm{cm}^{2}$ and of MEA 2 was $4 \times 10^{-5} \mathrm{~cm}^{3} / \mathrm{s} \cdot \mathrm{cm}^{2}$. However, MEA 7 damaged by carbon fibers from
GDLs had holes that developed very fast. Because of very high gas crossover $\left(160 \times 10^{-5} \mathrm{~cm}^{3} / \mathrm{s} \cdot \mathrm{cm}^{2}\right)$, the test was terminated only after $\sim 90$ hours of running (Figure 2).

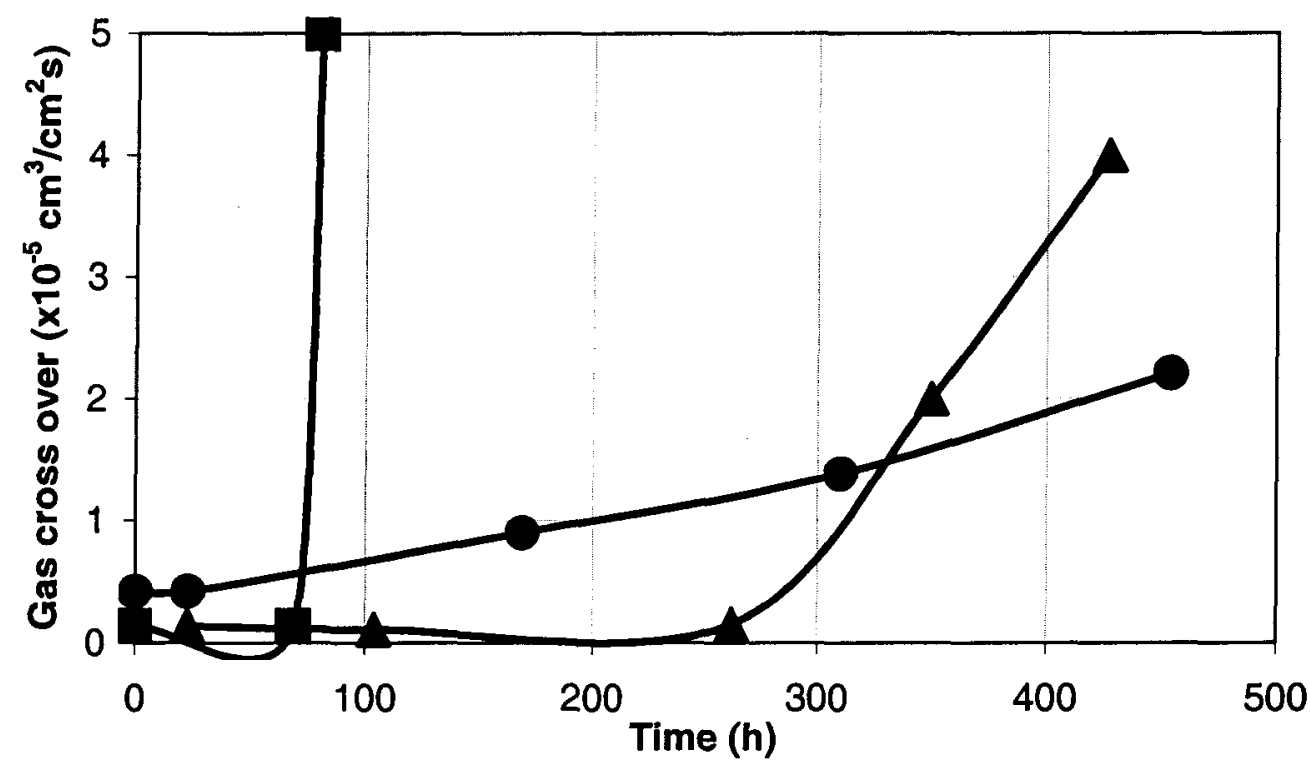

Figure 2: The gas crossovers of MEA s $1(\bullet), 2(\boldsymbol{\Delta})$ and MEA $7(\boldsymbol{\square})$ that failed durability screening tests. MEAs 1 and 2 developed gas crossover at the slower rate than MEA 7.

From these results, it can be noticed that membrane thickness (MEAs 1 and 2) did not affect MEA failure time. The membranes failed at almost the same time, although MEA 2 was $25 \%$ thicker than MEA 1.

MEA 8 with a $\mathrm{T} \mu \mathrm{m}$ thick membrane did not fail during the durability test although it had the same thickness as MEA 1 . It was processed by casting from polymer solution in lower alcohols and water. It appears that the reinforcement incorporated in the membrane improved the MEA durability. The gas crossover stayed constant during the entire accelerated life test.

MEAs 3, 4, 5, 6, 8 and MEA 9 have membranes prepared by polymer extrusion. Independent of the thickness, all these MEAs had unchanged gas crossover rates during durability tests. Comparing the gas crossovers of MEAs with the same thickness and different processing (MEA 2, 3, and 9), it can be observed that only MEA 2 with casted membrane failed the test. It seems that the membrane extrusion process is the most important factor contributing to the endurance. In fact, the molecular weight of polymers processed by extrusion is higher than the molecular weight of those processed by casting, creep of extruded polymers that occurs at slower rates extends membrane durability. Other MEA differences such as the catalyst loading, thickness, and composition of the micro diffusion layer may also have an effect on durability. However, these differences can most likely be observed only during longer tests. 

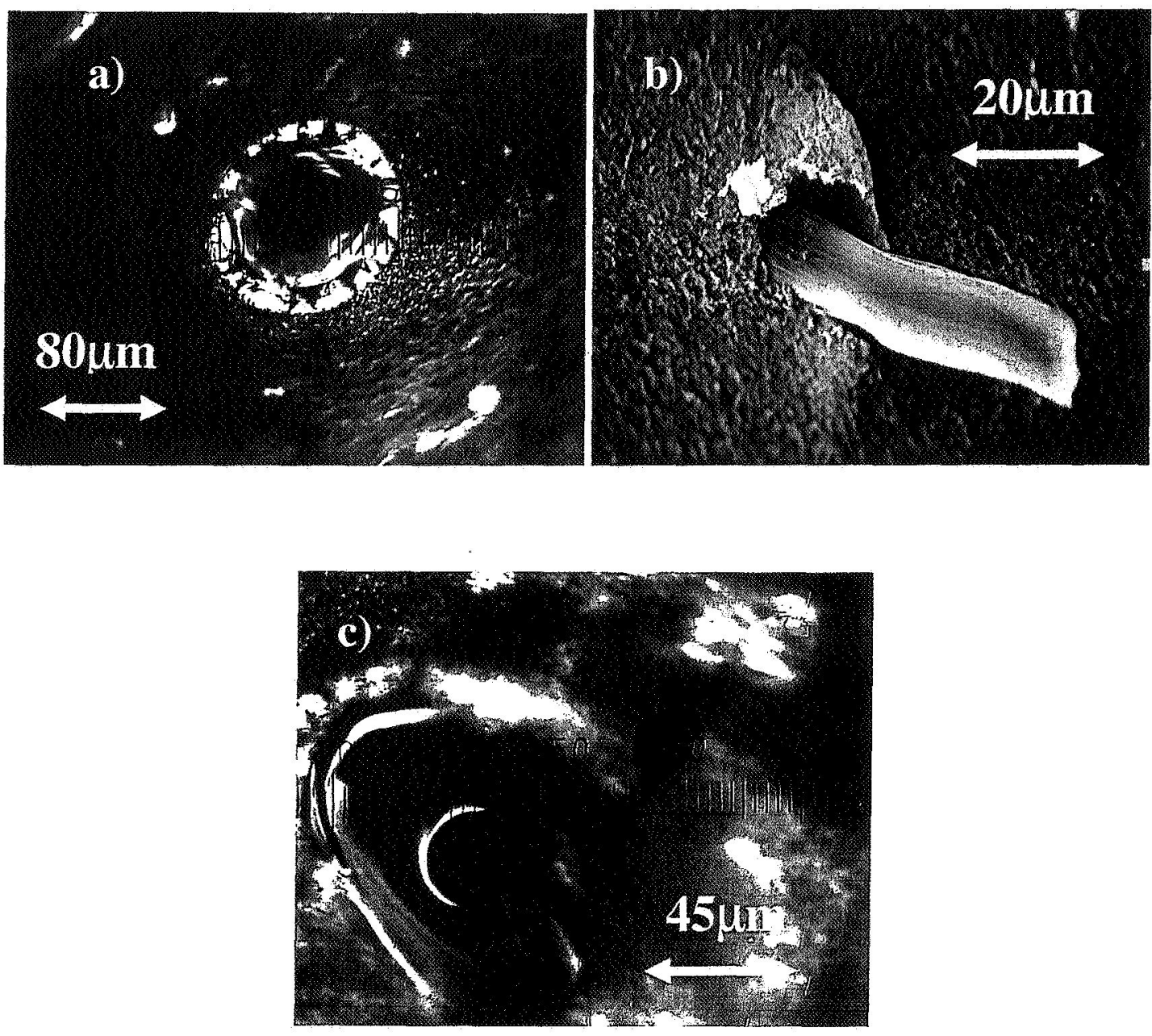

c)

Figure 3: Failure modes of MEAs: a hole in MEA 1 (optical micrograph a) is created by carbon fiber from GDL (scanning electron micrograph b); a hole in MEA 2 (optical micrograph c) is a typical appearance of "hot spot" failure mode in MEAs.

In Figure 3, the optical and scanning electron micrographs of MEA failure modes are presented. A hole created by carbon fiber in MEA 7 is shown in Fig. 3a, and the corresponding carbon fiber that caused formation of this hole is shown in Fig.3 b. The carbon fiber shown in the micrograph is penetrating through the micro diffusion layer of the cathode GDL. Since a short circuit was not identified in the stack with MEAs 7, it appears that carbon fibers at the MEA-GDL interface create higher compression spots that accelerate the polymer membrane creep. This may explain the mechanism of this failure. At the areas of higher compression, the membrane may be thinner and thus it has lower resistance. The lower resistance of the MEA results in a locally higher reaction rate. Higher reaction rate generates more heat that further accelerates the membrane creep. Therefore, the combination of higher compression, thinner membrane, and higher reaction rate, increases temperature locally that causes polymer electrolyte membrane to collapse at the contacts with carbon fibers faster.

The failure mechanism of membranes by holes formed due to "hot spots" is the objective of ongoing research at Teledyne Energy Systems.

\section{MEA performance}

Polarization curves of all MEAs tested in the 4-cell stack are presented in Figure 4. The curves represent the average stack potential. In low current regions ( $<$ $200 \mathrm{~mA} / \mathrm{cm}^{2}$ ), MEA performances were between 0.820 
and $0.840 \mathrm{mV}$. The maximum performance was achieved with MEA 9, while the minimum performance with MEA 6. The difference in the performance can be attributed to the cathode catalyst loading and the membrane thickness for MEA 9 and 6, respectively.
For the other MEAs, the performance was distributed within this $40 \mathrm{mV}$ range and depended on GDL type, electrocatalyst composition, catalyst loading, and membrane thickness.

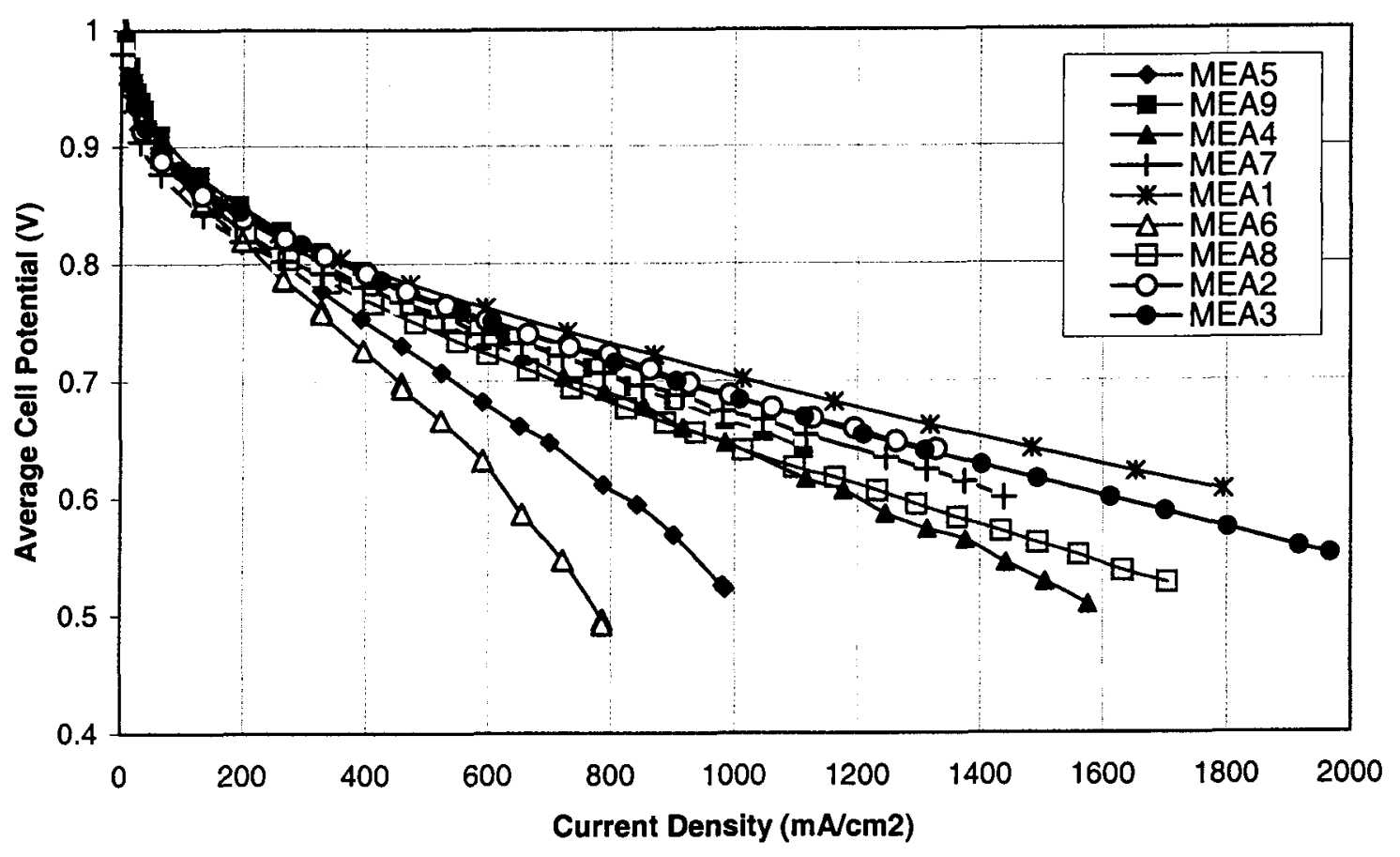

Figure 4: Polarization curves of MEAs selected for durability screening tests. The polarization curves are measured at nominal operating conditions.

In the high current region $\left(>400 \mathrm{~mA} / \mathrm{cm}^{2}\right)$, the MEA performance is predominately a function of membrane thickness. MEAs 1, 2 and 3 have almost identical performance up to $400 \mathrm{~mA} / \mathrm{cm}^{2}$ at nominal operating conditions (Figure 4). However, at accelerated conditions this range is extended to higher current values. In Figure 5, the steady state performances of these three MEAs are presented. Data were collected at accelerated conditions during durability tests. As shown in Fig. 5, the voltages of two thicker membranes (MEAs 2 and 3) are the same as the voltage of the thin MEA1. The reason for this behavior is probably the lower intrinsic permeability of the thicker membranes that compensates for their ohmic losses. 


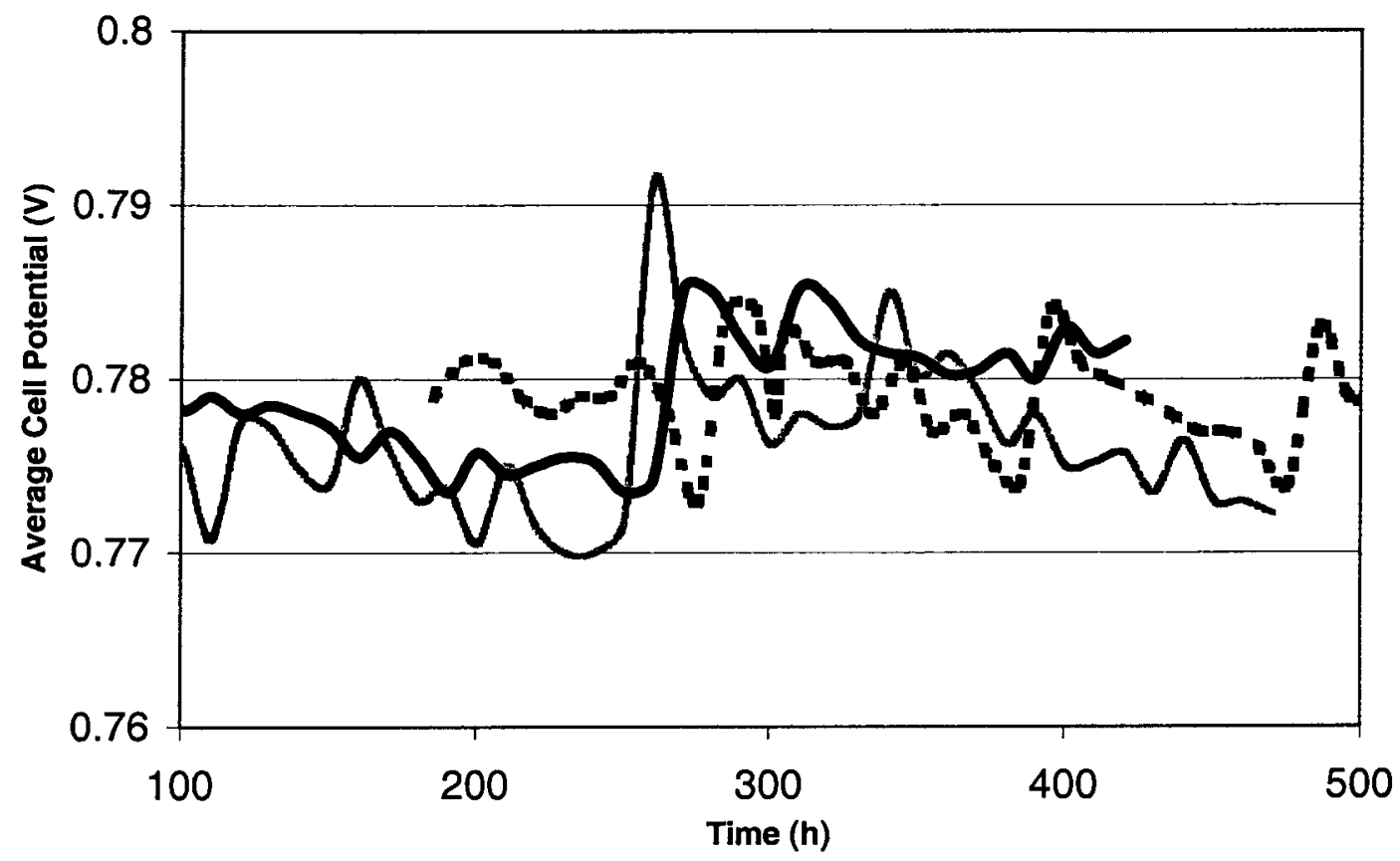

Figure 5: The steady state performance of three different MEAs tested at accelerated conditions: MEA 1(- - -), MEA $2(-)$, MEA 3(-) . All three MEAs have almost identical performance regardless thickness or preparation.

During the test run presented in Figure 5, MEAs 1 and 2 started to have gas-to-gas leaks. At the end of the test, gas crossovers were $2 \times 10^{-5}$ and $4 \times 10^{-5} \mathrm{~cm}^{3} / \mathrm{s} \cdot \mathrm{cm}^{2}$ for MEAs 1 and 2, respectively. However, these crossover rates were obviously too low to affect the stack performance. The potential variations presented in Fig. 5 are only the consequence of operating condition oscillations.

\section{Conclusions}

Durability of various MEAs was evaluated at accelerated operating conditions with hydrogen and oxygen. Three MEAs out of nine tested failed during the evaluation. Operating conditions other than temperature and humidification affect the MEA durability. If fuel cell current, gas stoichiometry, and pressure are selected properly, they can either cause the accelerated MEA failure or they can significantly extend the MEA life. Test results demonstrated that the type of membrane used for MEA preparation is essential factor for MEA durability. Casted membrane with reinforcement and extruded membranes passed the accelerated durability test. However, MEAs with casted membranes failed the test even though they had
To which degree gas crossover can increase without affecting stack performance is demonstrated with the performance results of MEA 1 summarized in Table 3. As an indicator of the crossover effect on stack performance, the voltage standard deviation was used. The standard deviation began to increase from 0 to \pm $12 \mathrm{mV}$ when crossover reached $114.5 \times 10^{-5} \mathrm{~cm}^{3} / \mathrm{s} \cdot \mathrm{cm}^{2}$. This potential deviation corresponds to the reactant pressure loss of $17.5 \%$. 


\section{$\underline{\text { Reference }}$}

1. D.R. Askeland, The Science and Engineering of materials, $2^{\text {nd }}$ ed, Chapman and Hall, New York, 1990,

p243.

2. J. Larminie and A.Dicks, Fuel Cell Systems

Explained, John Wiley \& Sons, Ltd., Chichester,

England, 2000, p.46.

Table 3: The gas crossover measured in a 4-cell stack assembled with MEA1. The gas crossover measurements are done with nitrogen at 5 psid. The average stack voltage is measured during a durability run.

\begin{tabular}{|l|l|l|l|}
\hline $\begin{array}{c}\text { Time } \\
\text { (h) }\end{array}$ & $\begin{array}{l}\text { Gas crossover } \\
\left(\mathbf{1 0} \mathbf{c m}^{\mathbf{3}} / \mathbf{s} \cdot \mathbf{c m}^{\mathbf{2}}\right)\end{array}$ & $\begin{array}{l}\text { Average stack } \\
\text { voltage } \\
(\mathbf{m V})\end{array}$ & $\begin{array}{l}\text { Cell voltage standard } \\
\text { deviation } \\
( \pm \mathbf{~ m V})\end{array}$ \\
\hline 0 & 0.8 & 730 & 0 \\
67 & 0.8 & 760 & 0 \\
414 & 4.6 & 780 & 0 \\
504 & 114.5 & 778 & 12 \\
545 & 131.8 & 775 & 20 \\
590 & 302.2 & 773 & 25 \\
663 & 1837.7 & 771 & 32 \\
\hline
\end{tabular}

